\section{$\$$ Research Square}

Preprints are preliminary reports that have not undergone peer review.

They should not be considered conclusive, used to inform clinical practice, or referenced by the media as validated information.

\title{
Cordycepin Combined with Doxorubicin Inhibits Triple-negative Breast Cancer by TNF Signaling Pathways
}

\section{Haichen Huang}

Fujian Agriculture and Forestry University

\section{Wenya Wu}

Fujian Agriculture and Forestry University

\section{Xiaomin Li}

Fujian Agriculture and Forestry University

Chengyi Liu

Fujian Agriculture and Forestry University

\section{Xiaoping Wu}

Fujian Agriculture and Forestry University

\section{Junli Zhang}

Tibet Academy of Agricultural and Animal Husbandry Sciences

Junsheng Fu ( $\nabla$ fujunsheng81@163.com )

Fujian Agriculture and Forestry University College of Life Science

\section{Research}

Keywords: triple-negative breast cancer, cordycepin, network pharmacology, TNF signaling pathway

Posted Date: May 7th, 2021

DOl: https://doi.org/10.21203/rs.3.rs-405699/v1

License: (c) (i) This work is licensed under a Creative Commons Attribution 4.0 International License. Read Full License 


\section{Abstract}

Background: Triple negative breast cancer (TNBC) is a subtype of breast cancer with high mortality and poor prognosis. This study was designed to explore the effect of cordycepin combined with doxorubicin against TNBC, and then explore the molecular mechanism of this process.

Methods: First of all, the effect of the combination of drugs on xenograft tumor and liver of mice was carried out to study the effect of combined drug on TNBC and its toxic effect on the body. Relevant genes are obtained through the GenBank and intersected, then the molecular mechanism is explored through GO, KEGG analysis $\triangle P P I$ network construction and other network pharmacological methods. The results of Network pharmacology through molecular docking, immunohistochemistry and other biological experiments to verify.

Results: The results show that the combination of drugs can significantly affect the size and weight of the xenograft tumor. The tumor weight of the control group is 3.57 times that of the combined group, and the effect is more obvious than that of the single medication. The liver status, liver index, AST and ALT index of mice were not significantly different $(P>0.05)$, indicating that the combination of drugs has no obvious toxic and side effects on the body. Through the above-mentioned series of network pharmacology methods, the final results we obtained focused on the TNF pathway and TNF-related targets such as MAP3K7, NFKB1, MAPK8 and so on. The results of molecular docking and biological verification were consistent with those of network pharmacology.

Conclusions: Cordycepin combined with doxorubicin can inhibit the growth of TNBC without obvious toxicity. The main molecular mechanism of action is to target and regulate the TNF pathway. This provides a new direction for clinical treatment of TNBC, which can effectively treat triple negative breast cancer and reduce the side effects of drugs.

\section{Background}

Breast cancer is the most common female malignant tumor in the world, ranking first among female cancers. Triple negative breast cancer (TNBC) refers to breast cancer in which estrogen receptor (ER), progesterone receptor (PR) and human epidermal growth factor receptor-2 (HER-2) are negative[1]. TNBC has the characteristics of high recurrence and metastasis, high mortality, and poor prognosis[2]. The current treatment of TNBC is mainly chemotherapy, and doxorubicin is one of the most effective chemotherapy drugs with strong activity characteristics[3]. But the side effects, drug resistance, high recurrence, and distant organ metastasis of the drugs are still the main reasons for the high mortality of TNBC[4,5]. In view of the huge defects in the treatment of TNBC, it is urgent to find new drugs with antitumor activity and no major body damage.

In recent years, the combination of drugs can improve the sensitivity of drugs, which has become a new method for the treatment of cancer, and will become the development trend of cancer treatment in the future[6]. Studies have found that natural products as one of the main sources of drugs, most of them 
have the characteristics of low toxicity and high safety. Some natural drugs have potential anti-tumor effects and have the potential to be used in clinical anti-tumor therapy. For example, Ginsenoside Rg3 combined with tumor statin 19 peptide can inhibit the proliferation of HepG2 cells, enhance the antitumor effect, reduce the dosage of single drug, and reduce the side effects of drugs[7]. The Polysaccharide from Ganoderma applanatum thermosensitive gel can significantly improve the antitumor effect of nanoparticle albumin-bound paclitaxel and alleviate the toxic and side effects caused by nanoparticle albumin-bound paclitaxel[8]. Develop compounds with anti-tumor effects from natural drugs, and achieve the effect of enhancing anti-tumor effects through drug combination, which provides a new direction for the research of anti-tumor drugs.

Cordycepin is an active substance isolated from Cordyceps militaris, which has anti-tumor, antibacterial, antiviral, immune regulation, free radical removal and other pharmacological effects[9]. Cordycepin has been found to inhibit the growth and metastasis of leukemia cells, lung cancer, liver cancer, prostate cancer, colon cancer and other tumor cells [10]. Pei Ma[11] showed that cordycepin could cause apoptosis of HeLa cells through interfering with RNA production, thus inhibiting the clinical cancer. Mousumi Tania[12] found that the treatment of cordycepin controlled SiHa and HeLa cervical cancer cell growth, increased apoptosis, and interfered with cell cycle, which proved that cordycepin has an obvious anti-tumor effect on cervical cancer cells. Secondly, cordycepin can be used as an auxiliary drug of antitumor drugs to improve the efficacy of the anti-tumor drugs themselves; and as a natural source of antitumor active ingredients, cordycepin has high safety[13]. According to the reports, cordycepin and platinum chemotherapeutic drugs (cisplatin) have significant anti proliferation, anti migration and pro apoptotic effects on human colon cancer cell line SW1116. The combination of cordycepin and cisplatin can significantly improve the curative effect, and the results suggest that cordycepin has a certain adjuvant effect on chemotherapy[14]. Then, according to the obtained experimental basis, we found that cordycepin can affect the growth, apoptosis and metastasis of human breast cancer cells through hedgehog pathway, which has significant anti-cancer effect and low toxicity. Therefore, in view of the poor prognosis and great damage to the body in the treatment of triple negative breast cancer and the antitumor activity and low toxicity of cordycepin.

In this study, the substance basis and mechanism of cordycepin combined with doxorubicin on TNBC were studied by drug combination and network pharmacology, provide theoretical basis for clinical treatment of triple negative breast cancer, and provide new ideas for the study of multi-target and multicomponent action mode of Chinese herbal compound.

\section{Methods}

\section{Reagents}

Human triple negative breast cancer cell line MDA-MB-231 was purchased from iCell Bioscience Inc. Cordycepin was purchased from Sigma(San Francisco,CA). Forty BALB / C nude mice,weighing 14-15g, aged 3-5 weeks, female, SPF grade) was obtained from Wu's laboratory animal 
Online http://www.fzzmsoft.com/xieli/[. RPMI 1640 medium, fetal bovine serum, green / streptomycin and Matrigel matrix were purchased from Gibco (Grand Island, NY); Trypsin was purchased from Beijing solarbio science $\lfloor$ technology co.,Itd Trypsin was purchased from Beijing solarbio science】technology co.,Itd; $4 \%$ paraformaldehyde, sodium chloride, potassium chloride, potassium dihydrogen phosphate, phenol, isopropanol and 30\% hydrogen peroxide were supplied by Sinopharm Chemical Reagent Co.,Ltd; ALT kit and AST Kit were purchased from Nanjing Jiancheng Bioengineering Institute; Trizol RNA Extraction Kit was purchased from invitrogen (Carlsbad, CA); Reverse transcription kit were SYBR greenqpcr kit was purchased from TransGen Biotech; Citrate buffer was purchased from Servicebio; Hematoxylin staining solution, neutral gum, secondary antibody: HRP labeled Goat anti rabbit secondary antibody and histochemical kit DAB chromogenic reagent was obtained from Beyotime Institute of Biotechnology.

\section{Cell culture}

Human triple negative breast cancer cells MDA-MB-231 were cultivated in RPMI 1640 medium after adding $10 \%$ fetal bovine serum, $1 \%$ cyanine / streptomycin, and mixing well and maintained in a constant temperature incubator containing $5 \% \mathrm{CO}_{2}$ at $37^{\circ} \mathrm{C}$.

\section{Xenograft tumor model}

MDA-MB-231 cells in the logarithmic growth phase were made into a suspension and injected into nude mice subcutaneously. When they grew to about $100 \mathrm{~mm}^{3}$, they were divided into four groups:Control group $\left(\mathrm{H}_{2} \mathrm{O}+0.9 \% \mathrm{NaCl}\right)$ and Cordycepin group $(20 \mathrm{mg} / \mathrm{kg})$ ), Doxorubicin group $(5 \mathrm{mg} / \mathrm{kg})$ and Combination group (DOX $(5 \mathrm{mg} / \mathrm{kg})+\mathrm{COR}(20 \mathrm{mg} / \mathrm{kg})$ ). The drug was administered once a week for 16 days, then fast but can't help water. After 24 hours, blood was taken from the eyeball and centrifuged to obtain serum. Quickly remove the tumor and liver, and detect various physiological indicators.

\section{Cytotoxicity analysis}

During the experiment, we measured and recorded the weight of nude mice every day, while observing the growth condition of the mice such as eating and activity. After the mice were dissected and taken out of the liver, they were quickly washed with normal saline to remove the excess blood. After the excess water was drained, the liver of the mice was observed for color, weighed, and the liver index was measured. Operate the serum in accordance with the ALT and AST test kit instructions, draw the standard curve of ALT and AST, test the OD value of the sample, and calculate.

\section{Prediction of revelant targets}

We collected the basis information and predicted targets of cordycepin and doxorubicin through databases(Table 1) such as PubChem, SEA SearchServer, Superpred and GeneCards. Use the Uniprot database to search for the protein name of the drug and obtain the gene name corresponding to the predicted target, and then obtain the breast cancer-related target gene in the Gene Cards database. Use the "SUMIF" function in Excel to intersect the related target genes and candidate target genes of cordycepin and doxorubicin. The three common target genes are used as potential targets for the 
combination of cordycepin and doxorubicin for anti-breast cancer. OmicShare draws a Venn diagram of three common targets.

Table 1

database

\begin{tabular}{|ll|}
\hline Database name & Web sites \\
\hline Pub Chem & http://pubchem.ncbi.nlm.nih.gov \\
\hline Uniprot & https://www.uniprot.org/ \\
\hline OmicShare & https://www.omicshare.com/tools/ \\
String & https://string-db.org/ \\
SEA & http://sea.bkslab.org/ \\
Bingding DB & http://www.bindingdb.org/bind/index.jsp \\
\hline SuperPred & http://prediction.charite.de/index.php/ \\
\hline Gene Cards & https://www.genecards.org/ \\
\hline Pubchem CID & https://pubchem.ncbi.nlm.nih.gov/ \\
\hline PDB & http://www.rcsb.org/ \\
\hline
\end{tabular}

\section{Protein interaction analysis}

We imported the potential targets of cordycepin combined doxorubicin against triple-negative breast cancer in string online detabase(Table 1) and set "Homo sapiens" as the species to search the target protein interaction relationship. Use the Network Analyzer tool in Cytoscape 3.6.0 to analyze the network, and classify it with Degree and Betweenness centrality. The PPI protein interaction network diagram was drawn by Cytoscape 3.6.0.

\section{GO analysis and pathway enrichment analysis of KEGG}

Go and KEGG of 76 intersection targets were analyzed in David database, and $P<0.01$ was used as the screening condition for statistical difference. The obtained GO term was made into bar graph, in which blue was the go biological process related to apoptosis, red was related to proliferation, and black was the biological process related to migration. KEGG analysis used $\mathrm{P}<0.01$ as the screening condition of statistical difference to obtain the top 20 pathways and draw the bubble chart.

\section{Construction of compound-pathway-target network}

Analyze the pathways that are most likely to be related to the treatment of breast cancer by combining the literature to find out the targets enriched in these pathways in the treatment of breast cancer by cordycepin combined with doxorubicin, and use the analysis results to predict and draw a "complex 
pathway-target" network diagram of the effect of cordycepin combined with doxorubicin against breast cancer.

\section{Molecular docking}

In order to study potential binding mode between cordycepin $\rrbracket$ doxorubicin and relevant proteins of the pathways with the most significant enrichment analysis. We use Auto Dock 4.2 for molecular docking. Then, the auto grid of auto dock 4.2 was used to calculate the relevant energy, and the region search and docking were carried out. PyMOL 2.3 was used to present the conformation of the compound with the best docking structure.

\section{Fluorescence quantitative PCR}

Trizol reagent was added to the xenograft tumor tissue of nude mice after grinding; according to the requirements of reverse transcription kit of TransScript All-in-one First-Strand cDNA Synthesis SuperMix for QPCR, the sample concentration was diluted to $500 \mathrm{ng} / \mu \mathrm{L}$ to synthesize single-stranded CDNA. Primer 5 was used to design the primers needed for fluorescence quantitative PCR and synthesized by Shanghai Shenggong Bioengineering Co., Ltd. Dilute the cDNA by 5 times, follow the instructions of the TransStart Top Green qRCR SuperMix kit, and use cDNA as a template for amplification. PCR reaction conditions: denaturation $\left(94^{\circ} \mathrm{C}, 5 \mathrm{~s}\right)$, annealing $\left(58^{\circ} \mathrm{C}, 30 \mathrm{~s}\right)$, extension $\left(60^{\circ} \mathrm{C}, 5 \mathrm{~s}\right)$, and perform 40 cycles. According to the $2^{-\triangle \triangle C T}$ method for relative quantitative analysis of the results.

\section{Immunofluorescence assay}

The slices were rinsed with PBS and fixed with 10\% Goat Serum for 30-60 min. After adding caspase-3 antibody, incubate overnight in a humid chamber at $4^{\circ} \mathrm{C}$, rinse with PBS twice, add HRP-labeled goat antirabbit secondary antibody, incubate at $37^{\circ} \mathrm{C}$ for $30 \mathrm{~min}$, then drop $S A B C$ and ncubate in at $37^{\circ} \mathrm{C}$ for 30 min, aspirate the SABC, add DAB solution and staining ends when red-brown substances appear. Rinse twice with PBS, stain with hematoxylin for 10 minutes, differentiate the slices in $1 \%$ hydrochloric acid alcohol for a few seconds, place in $1 \%$ ammonia water for 10 minutes, observe and take pictures under a microscope.

\section{Statistical analysis}

The data are processed by SPSS 23.0 statistical software package, and the measurement data are expressed as "mean \pm standard deviation $(x \pm s)$ ", using $t$ test, and $P<0.05$ indicates that there is a significant difference between groups.

\section{Result}

\section{Cordycepin combined with Doxorubicin inhibited the growth of xenograft tumor}


After dissecting the nude mice, the morphology of the xenograft tumor in nude mice was obtained, and the curative effect of combined therapy on the xenograft tumor in nude mice was observed intuitively. It can be seen that the order of tumor size is combination group < cordycepin group < doxorubicin group $<$ control group (Fig. 1A).

Secondly, the tumor growth curve was drawn using the tumor volume data recorded during the experiment (Fig. 1B). It can be seen that the growth curve of xenograft tumor in each treatment group showed an upward trend over time; On the 8th and 16th days of growth, there were significant differences $(P<0.05)$ and extremely significant differences $(P<0.01)$ between the control group and the other three groups, respectively. The final volume in descending order was the control group, cordycepin group, doxorubicin group. The volume of xenograft tumor in control group was 3.58 times of that in combination group.

According to the tumor volume recorded in cordycepin group, doxorubicin group and combination group, the tumor inhibition rate of the corresponding group to MDA-MB-231 cell xenograft tumor was calculated, which were $65.86 \% \pm 13.88$ and $59.19 \% \pm 15.30$ and $79.53 \% \pm 12.63$, respectively (Fig. 1B). The combination group had the highest tumor inhibition rate, which was significantly different from the doxorubicin group $(P<0.05)$, and was extremely significantly different from the cordycepin group $(P<$ $0.01)$. Here, we found an obvious trend that the order of tumor growth inhibition is doxorubicin group < cordycepin group < combination group.

In order to intuitively see the effect of the combined treatment on the xenograft tumor in nude mice, we dissected the xenograft tumor and took photos, and recorded the weight of each group of xenograft tumor. It can be seen that the order of tumor size was combination group < cordycepin group < doxorubicin group < control group (Fig. 1C).

The weight measurement results (Fig. 1D) are further analyzed, the four groups of xenograft tumor weight order is combination group < cordycepin group < doxorubicin group < control group, the tumor weight of the control group was 3.57 times that of the combination group. There were significant differences between the three treatment groups and the control group $(P<0.01)$, and there were extremely significant differences between the combination group and cordycepin, the combination group and the doxorubicin group $(P<0.01)$.

The above results show that cordycepin combined with doxorubicin can significantly inhibit triplenegative breast cancer, and the combined drug effect is more obvious than the single drug effect.

\section{Cell Cytotoxicity Assay of Cordycepin combined with Doxorubicin}

In order to understand the effects of drug combination on the body when it exerts its anti-tumor effect, we observed the mental status, body weight and liver function of nude mice. 
During the experiment, the physiological state of each group of animals was observed every day, and the body weight was weighed regularly. From the observation of physiological phenomena, the animals in each experimental group showed no abnormalities in eating, mentality, and exercise. The results in Fig. 2A showed that there was no significant difference in body weight between the combination group, cordycepin group, doxorubicin group and control group $(P>0.05)$. There was no significant change in body weight, which proved that the amount of reagents in each group was appropriate, and the combination medication had no obvious side effects on mice.

After the nude mice were dissected, their livers were quickly taken out and photographed. The results (Fig. 2B) showed that, compared with the control group, the liver color and texture of the three experimental treatment groups were similar to those of the control group. The livers of the four groups were rosy, uniform and elastic.that the three experimental treatment groups were compared with the control group.

According to the weight of nude mice in each treatment group, the liver index of each treatment group was calculated (Fig. 2C-D). The liver index of the cordycepin group, the doxorubicin group and the combination group was close to that of the control group, and there was no significant difference between the four groups $(P>0.05)$.

By detecting the contents of ALT and AST in serum (Fig. 2E-F), we found that compared with the control group, the serum ALT and AST contents of the three experimental groups had no significant change, and there was no significant difference compared with the control group $(P>0.05)$.

The above results indicate that cordycepin, doxorubicin and the combination of drugs did not cause significant damage to the liver of nude mice, verifying that cordycepin combined with doxorubicin has low toxicity in the treatment of triple-negative breast cancer.

\section{Screening of common targets in cordycepin $\square$ doxorubicin and TNBC}

In order to explore the mechanism of drug combination, we need to know the related or potential targets of diseases and drugs in the whole process of network pharmacology.

After database collection, merging, and removal, the final targets of cordycepin and doxorubicin were 147 and 2,469 respectively, and 13134 targets related to triple-negative breast cancer were searched. The Venn diagram (Fig. 3A) shows that there are 76 common targets for cordycepin, doxorubicin and triplenegative breast cancer, indicating that there are 76 potential targets for cordycepin combined with doxorubicin in the treatment of triple-negative breast cancer.

\section{Construction of PPI network for target interaction of drug combination}


After obtaining the target genes mentioned above, we need to analyze the relationship between the two or more genes. Import 76 potential target genes into the String database to construct a PPI network (Fig. 3B). The results show that the PPI network includes 76 nodes and 532 edges. The circular node represents the target gene, and the straight line between the nodes indicates that there is interaction between the two proteins. In the graph, the larger the node and the darker the color, the higher the degree value of the corresponding target gene, otherwise. The targets with larger nodes are CASP3, MAPK8, MMP9, IL2, NFKB1. It is proved that these genes play an important interaction in the entire protein network, which may have a key significance in the mechanism of cordycepin combined with doxorubicin in the anti-triple-negative breast cancer.

\section{GO Analysis of Targets of Combination Drugs}

After understanding the relationship between genes, we need to understand their role in the related biological processes in which they participate. Go BP analysis was performed on 76 intersecting targets in David database, and then $12 \mathrm{GO}$ terms with $\mathrm{P}<0.01$ were selected as the bar graph. Blue is the go biological process related to apoptosis, red is related to proliferation, and black is the biological process related to migration. The results (Fig. 4A) showed that in the first 12 biological processes, the target genes were mainly concentrated in the biological processes related to apoptosis, such as "negative regulation of apoptotic process", "apoptotic process", "positive regulation of apoptotic process", and "regulation of apoptotic process"; the biological processes related to Proliferation mainly focus on "regulation of cell Proliferation", "positive regulation of activated T cell proliferation" and "negative regulation of cell proliferation"; while in migration, it focuses on the "biological process of leucocyte migration".

These results indicate that cordycepin combined with doxorubicin can induce apoptosis of breast cancer cells and inhibit the proliferation and migration of breast cancer cells by acting on multiple targets, so as to achieve the treatment of triple negative breast cancer.

\section{KEGG enrichment analysis of target of Combination Drugs}

Through the KEGG database, the pathway enrichment analysis of the target of cordycepin combined with doxorubicin for anti-breast cancer was carried out, and the relevant signal pathway was obtained with $P<$ 0.01 as the screening condition. According to the degree of concentration, the leading pathways are TNF signaling pathway, MicroRNA in cancer, IL-17 signaling pathway, AGE-RAGE signaling pathway in diabetic complications, apoptosis,Epithelial cell signaling in Helicobacter pylori infection and other tumor related signaling pathways. We measured the enrichment of KEGG according to $p$ value and and found that the TNF signal pathway was the most significant of the enriched signal pathways.Comprehensive literature reports, TNF signaling pathway is mainly involved in tumor migration and apoptosis process ${ }^{[15,16]}$, indicating that cordycepin combined with doxorubicin can inhibit breast cancer growth by regulating multiple pathways, among which TNF signaling pathway inhibits tumor migration and apoptosis is the master. 


\section{Analysis of the target pathway network of Combination Drugs}

Using KEGG's pathway enrichment analysis to obtain the relevant targets of the combined drug action, combined with relevant literature theoretical reports, draw the final pathway map (Fig. 5). The figure shows that cordycepin combined with doxorubicin can regulate the MAPK signaling pathway, NF-KB, IL17 signaling pathways and apoptosis pathways ultimately by acting on multiple factors in the TNF signaling pathway, such as MAP3K7, NFKB1, MAPK8, CFLAR, CASP3, CCL2, CXCL1, JUNB, MMP3, MMP9, ICAM1. The signaling pathways ultimately act on breast cancer cell apoptosis and metastasis, suggesting that the anti-breast cancer mechanism of cordycepin combined with doxorubicin is importantly related to the TNF signaling pathway.

\section{Analysis of molecular docking between cordycepin, doxorubicin and target}

According to the results obtained from the above network pharmacological analysis, cordycepin and doxorubicin are used as ligands, and 11 targets enriched in the TNF signaling pathway are used as receptors. Auto Dock 4.2 software is used for molecular docking. Among the proteins that bind cordycepin and doxorubicin, the proteins with BE $\leq-5 \mathrm{KJ} / \mathrm{mol}$ include: CFLAR, NFKB1, CASP3, MAP3K7, JCAM1, MAPK8, MMP3 and CFLAR, NFKB1, CASP3, MAP3K7, JCAM1, MAPK8, MMP3, MMP9, CXCL1, MAPK8, respectively, the proteins that bind cordycepin and doxorubicin with the smallest binding energy are CFLAR and MMP3 proteins, respectively.

From the point of view of the inhibition constant, cordycepin has the strongest inhibitory strength on CFLAR protein $(I C=6.77 \mu \mathrm{mol} / \mathrm{L})$, and doxorubicin has the largest inhibitory strength on MMP3 $(\mathrm{IC}=0.29$ $\mu \mathrm{mol} / \mathrm{L})$.

In summary, it can be seen that, cordycepin and doxorubicin have shown varying degrees of regulation on different proteins in the TNF signaling pathway, while they have consistently high binding activity and strong inhibitory effects on the target of CFLAR. This indicates that cordycepin combined with doxorubicin has a stronger regulatory effect on the TNF signaling pathway in breast cancer than when it has a single action, showing a synergistic or additive effect, suggesting that cordycepin combined with doxorubicin can induce TNF signaling pathway Apoptosis of breast cancer.

\section{Analysis of the interaction between cordycepin, doxorubicin and key proteins}

According to the results of molecular docking, three proteins with molecular docking Binding Energy less than $-5 \mathrm{KJ} / \mathrm{mol}$ and Inhib Constant less than $1 \mathrm{mmol} / \mathrm{L}$ were screened out, and PyMOL software was used for visual analysis. Figure $6 \mathrm{~A}$ shows that cordycepin and doxorubicin can stably dock into the receptor active pocket of CFLAR. Cordycepin has H-bonding through amino acid residues GLN374, GLU398, GLN468, PI-bonding through amino acid residues GLN450, ARG337, HIS334, GLN319, and H-PI 
compound bond through ASN392 residues(Fig. 6A a-b). Doxorubicin has $\mathrm{H}$-bonding through amino acid residues GLU315 and LYS305, PI-bonding through amino acid residue TYR319, and H-PI compound bonding through amino-acid residues ASN392, ASN393, GLN374, and THR375(Fig. 6A c-d).

Figure 6B shows the best composite conformation of cordycepin and doxorubicin for docking with MAP3K7 protein, respectively. Cordycepin mainly interacts with amino acid residue PRO292 through Hbonding, and through ALA127, TYR124, PHE487 residues to form PI bond, and interacts with ASP483, PHE484 residues form the $\mathrm{H}$-PI compound bond(Fig. 6B a-b); Doxorubicin forms two $\mathrm{H}$ bonds through PR0292 and TYR290, and forms PI bonds with ASP483 and ALA127(Fig. 6B c-d). These interactions are the main effects of cordycepin and doxorubicin stably docking to the active pocket of MAP3K7; Fig. 6C shows the best composite conformation of cordycepin and doxorubicin for docking with MMP3 protein.

Cordycepin mainly interacts with VAL198, GLU202, LEU22 residues through H bonds, and HIS224 forms H-PI composite bonds(Fig. 6C a-b); Doxorubicin forms two hydrogen bonds with GLU125 and GLU118, forms a PI bond with ARG134, and forms an H-PI compound bond with PHE132(Fig. 6C a-b). These interactions are the main function of cordycepin and doxorubicin which can stably dock into the active pocket of MMP3.

This indicates that cordycepin and doxorubicin can interact with different residues of the active site of the target protein to form a stable complex, the corresponding targets in the TNF signaling pathway are adjusted to varying degrees, and finally the growth of triple-negative breast cancer is inhibited, which is consistent with the analysis of network pharmacology.

\section{The effect of drug combination on the expression of key protein targets}

Based on the results of molecular docking, the changes in the expression of enriched target genes in the TNF signaling pathway when cordycepin combined with doxorubicin act on breast cancer were detected. Fluorescence quantitative detection results(Fig. 7A-B) showed that, compared with the control group, cordycepin combined with doxorubicin down-regulated the expression of ICAM1, JUNB, CXCL1, CCL2, MMP3, CFLAR, NFKB1, MMP9, MAP3K7 and other factors, and there was a significant difference $(P<$ 0.05). Among them, the most obvious down-regulation of cordycepin was the JUNB gene, which was down-regulated 2.54 times, and doxorubicin inhibited CXCL1 most significantly, down-regulated 4.08 times. At the same time, compared with the control group, the combination of cordycepin and doxorubicin up-regulated the expression of CASP3 gene, which was 3.94 times and 2.99 times that of the cordycepin combined with doxorubicin group, respectively, with extremely significant differences $(P<0.01)$.

After immunohistochemical detection, it was found that, consistent with the fluorescence quantitative results, the combination of cordycepin and doxorubicin also significantly increased the protein expression of CASP3 in breast cancer tissues. 
Biological verification results( Fig. 7C) show that cordycepin and doxorubicin modulate multiple target genes in the TNF signaling pathway in vivo to varying degrees, showing synergistic or additive effects, which is consistent with the results of molecular docking, indicating that cordycepin is combined Doxorubicin inhibits the growth of breast cancer by regulating multiple targets and multiple pathways. The regulation of TNF signaling pathway is one of the mechanisms of action of cordycepin combined with doxorubicin to resist breast cancer.

\section{Discussion}

Cordycepin has multiple pharmacological effects, including antibacterial, anti-inflammatory, anti-tumor, anti-viral, and improving human immunity. It has great development value and broad market prospects in food, medicine, and health products ${ }^{[13]}$. A large number of experimental studies have confirmed that cordycepin has anti-tumor effect, and cordycepin has been widely used to solve the problems caused by cancer treatment For example, cordycepin can affect the proliferation, migration, invasion, cell cycle and apoptosis of lung cancer, and solve the problem of poor drug resistance of gefitinib and afatinib in the treatment of lung cancer ${ }^{[17]}$. Cordycepin treatment makes breast cancer cells sensitive to radiation through Nrf2 / HO-1 / ROS axis, and solves the situation that radiotherapy is ineffective due to the radioresistance of cancer cells ${ }^{[18]}$. Cordycepin induces apoptosis of human bladder cancer T24 cells by activating exogenous and intrinsic apoptotic pathways and ROS dependent inactivation of PI3K / Akt signal ${ }^{[19]}$. At present, chemotherapy is the main treatment for breast cancer, but the clinical chemotherapy drugs have the disadvantages of poor drug resistance, high toxicity and many adverse reactions, so the clinical treatment gradually starts to adopt the combination of drugs, in order to improve the curative effect and reduce the drug resistance and toxicity to the body ${ }^{[20]}$. Cordycepin as an antitumor drug development direction, and cordyceps has a high natural safety, is also widely used in the treatment of breast cancer. For example, cordycepin combined with gemcitabine can significantly inhibit the growth of breast cancer cells, promote cell apoptosis, and thus inhibit breast cancer ${ }^{[20]}$. Therefore, in this experiment, cordycepin and doxorubicin were used in combination, and their effects on mice were studied to explore the effect of cordycepin combined with doxorubicin on triple negative breast cancer.

Through network pharmacology, we can study the complexity of diseases and traditional Chinese medicine ingredients, and provide a scientific method and idea for the study of complex components and pharmacological mechanism of traditional Chinese medicine. Rebecca Klee ${ }^{[21]}$ studied the mechanism of drug action on epilepsy through network pharmacology, and found that epilepsy could not be effectively treated by single drug due to its complex pathogenesis, and proposed the strategy of evaluating the rational choice drug combinations on epilepsy. Huiping Liu ${ }^{[22]}$ discovered some signaling pathways of XYP acting on infertility and some biological processes that may influence anovulatory infertility with XYP through network pharmacology, providing ideas for the treatment of infertility.

In this experiment, we infer that the enrichment of targets is relatively significant through network pharmacology, including TNF signaling pathway, microRNAs, IL-17 signaling pathway, AGE-RAGE 
signaling pathway and apoptosis pathway. According to the literature reports, TNF - a can trigger activation of NF - $\mathrm{K}$ B and MAPK pathway and other multiple pathways ${ }^{[23]}$. NF - $\mathrm{K} \mathrm{B}$ is a nuclear transcription factor in eukaryotic cells, which plays an important role in the proliferation, differentiation, invasion and metastasis of tumor cells, Activation of NF - $\mathrm{K} B$ has been observed in a variety of human malignant tumors ${ }^{[24]}$. The activation of caspase induces cell apoptosis, thus activating the upstream promoter caspase8, and directly activating the downstream Caspase 3,6 and 7 through the cascade amplification reaction, and the activated Caspase3 can cleave and repair DNA molecules, apoptosis inhibitory effector protein, extracellular matrix protein and cytoskeleton protein, thus promoting the apoptosis of tumor cells ${ }^{[25]}$. Abnormal expression of miRNAs is closely related to tumor proliferation, invasion and metastasis, angiogenesis, cell resistance and signal pathway ${ }^{[26]}$. Fabrice benchtrit inhibited the growth of mast cell tumor P815 and plasma cell tumor j558I through IL-17, and inferred that IL-17 participated in the anti-tumor process through $\mathrm{T}_{\text {cells }}{ }^{[27]}$. Cordycepin can inhibit the activation of MAPK induced by TNF signal, inhibit the expression of cflar, regulate the CASP family and MMP family, thus inhibiting the proliferation and metastasis of tumor, inducing apoptosis and autophagy of breast cancer. and research on these targets shows that Cordycepin can inhibit the activation of MAPK induced by TNF signal, inhibit the expression of CFLAR, regulate the CASP family and MMP family, thereby inhibiting tumor proliferation, metastasis, and inducing breast cancer apoptosis and autophagy ${ }^{[28-30]}$.

This experiment proved that cordycepin combined with doxorubicin has obvious anti-tumor effect and no obvious toxicity on triple negative breast cancer, and the mechanism of combined therapy was explored through network pharmacology. This provides a theoretical and practical basis for the development of cordycepin related products and drugs in the later stage, and provides a new idea for clinical medication of TNBC.

\section{Conclusion}

In summary, cordycepin combined with doxorubicin can inhibit the growth of breast cancer without obvious side effects, while the mechanism of cordycepin combined with doxorubicin against triplenegative breast cancer is mainly through the regulation of TNF signaling pathways, among which 11 key targets such as CFLAR, NFKB1, MAP3K7, MAPK8, CASP3, ICAM1, MMP3, MMP9, JUNB, CXCL1, CCL2 play an important role.

\section{Abbreviations}

TNBC (Triple negative breast cancer), GO(Gene Ontology), KEGG(Kyoto Encyclopedia of Genes and Genomes) , ER (estrogen receptor), PR (progesterone receptor) , HER-2 (human epidermal growth factor receptor-2), COR (Cordycepin ), DOX (Doxorubicin), CON group (control group).

\section{Declarations}




\section{Funding}

This research was supported by the National Natural Science Foundation of China (81503187) and innovative research of characteristic edible fungi germplasm resources and integrated demonstration of key techniques for strain breeding (XZ202001ZY0041N) .

\section{Availability of data and materials}

All data in this current study are available from the corresponding author upon reasonable request.

\section{Ethics approval and consent to participate}

All the animal studies were approved by Fujian Agriculture and Forestry University Ethics Committee.

\section{Competing interests}

The authors declare that they have no competing interests.

\section{Consent for publication}

All authors reached an agreement to publish the study in this journal.

\section{Authors' contributions}

Haichen Huang, Wenya Wu, Xiaomin LI, Chengyi Liu, Xiaoping Wu, Junli Zhang and Junsheng Fu conceived and designed the experiments. Haichen Huang, Wenya Wu performed the experiments. Haichen Huang, Wenya Wu, Xiaoping Wu, Junli Zhang and Junsheng Fu made substantial contributions to the interpretation of data. Haichen Huang, Wenya Wu, Xiaomin LI, Chengyi Liu wrote the first draft of the manuscript. Haichen Huang, Wenya Wu, Xiaomin LI, Chengyi Liu revised the draft and approved the version submitted. All authors have read and agreed to the published version of the manuscript.

\section{Author details}

1 College of Life Sciences, Fujian Agriculture and Forestry University, Fuzhou, Fujian, 350002, China.

2 Mycological Research Center, Fujian Agriculture and Forestry University, Fuzhou, Fujian, 350002, China.

3 Tibet Academy of Agricultural and Animal Husbandry Sciences, Lhasa, Tibet, 850000, China. 
tHaichen Huang and Wenya Wu contributed equally to this work.

*Corresponding author. E-mail: fujunsheng81@163.com

\section{Acknowledgements}

Thanks to other members of the laboratory for their help.

\section{References}

1. Bryan B B , Schnitt S J , Collins L C . Ductal carcinoma in situ with basal-like phenotype: a possible precursor to invasive basal-like breast cancer. Mod Pathol, 2006, 19(5):617-621.

2. Mirzania M , Safaee S R, Shahi F, et al. Treatment Outcomes and Clinicopathologic Characteristics of Triple-Negative Breast Cancer: A Report from Cancer Institute of Iran. Int J Hematol Oncol Stem Cell Res, 2017, 11(1):37-42.

3. Wang JH, Shi M, Ling R, et al. Adjuvant chemotherapy and radiotherapy in triple-negative breast carcinoma: A prospective randomized controlled multi-center trial. 2011, 100(2):200-204.

4. Carvalho F S , Burgeiro A, Garcia R , et al. Doxorubicin-Induced Cardiotoxicity: From Bioenergetic Failure and Cell Death to Cardiomyopathy. Medicinal Research Reviews, 2014, 34(1):106-135.

5. Ni C, Li T, Wu ZH, Hu XC. Progress on chemotherapy for triple negative breast cancer . China Oncology, 2014,24(04):316-320.

6. Cohen M H, W.G.A., Sridhara R, et al. United States Food and Drug Administration Drug Approval summary: Gefitinib (ZD1839; Iressa) tablets. Clinical Cancer Research, 2004, 10(4):1212-8.

7. Yi TH, Wu YM, Liu R, et al. Effects and mechanism of ginsenoside Rg3 combined with tumstatin 19 peptide on apoptosis of HepG2 cells in liver cancer,2020,43(07):575-582.

8. Tang L , Zhu ZF , Cao LP, et al. Thermosensitive gel of polysaccharide from Ganoderma applanatum combined with paclitaxel for mice with $4 \mathrm{~T} 1$ breast cancer. China journal of Chinese materia medica, 2020, 45(11):2533-2539.

9. Wang CM, LI L, Yang QZ, et al. The Research Progress of Clinical Application of Cordycepin-the Main Active Constituents of Cordceps Militaris. Guangzhou chemical industry, 2009. 37(05): 42-43.

10. Wang Z, Wu X, Liu JL, et al. Advances in research on Antitumor Activities of Cordycepin, Chinese Journal of pharmacy, 2015, 50 (16): 1365-1368

11. Ma $P, X u L$, Wang L , et al. Molecular Detection of Cordycepin-Induced HeLa Cell Apoptosis with Surface-Enhanced Raman Spectroscopy. Applied Sciences, 2019, 9(19).

12. Tania M , Shawon J , Saif K, et al. Cordycepin Downregulates Cdk-2 to Interfere with Cell Cycle and Increases Apoptosis by Generating ROS in Cervical Cancer Cells: in vitro and in silico Study. Current cancer drug targets, 2019, 19(2):152-159. 
13. Wang CW, Wang Z, Wang JF, et al. Research progress of cordycepin. Fujian agricultural science and technology, 2019 (2): 66-70

14. Cai W, Ye Q, Tang L, et al., The effect of cordycepin alone or combined with chemotherapy on the proliferation, migration and apoptosis induction of colon cancer cells in vitro. Chinese Journal of Clinicians (Electronic Edition). 2011. 5(14): 4048-4054.

15. Kai $S$, Shuyuan $Y$, Chawnshang $C$, et al. TNF signaling mediates an enzalutamide-induced metastatic phenotype of prostate cancer and microenvironment cell co-cultures. Oncotarget, 2015, 6(28):25726-25740.

16. Lee YS, Dutta A . MicroRNAs in cancer. Annual Review of Pathology, 2009, 4(1):199-227.

17. vila-Carrasco L , Majano P, JA Sánchez-Toméro, et al. Natural Plants Compounds as Modulators of Epithelial-to-Mesenchymal Transition. Frontiers in Pharmacology, 2019, 10:715-.

18. Dong JL,Li Y,Xiao HW, et al. Cordycepin sensitizes breast cancer cells toward irradiation through elevating ROS production involving Nrf2. Toxicology and applied pharmacology,2019,364.

19. Yoo H. Cordycepin inhibits human ovarian cancer by inducing autophagy and apoptosis through Dickkopf-related protein $1 / \beta$-catenin signaling. Journal of Acupuncture and Meridian Studies,2020,13(2).

20. Hua Fan, Yongjun Zhu, Yongfeng Zhao, Effect of cordycepin combined with Gemcitabine on apoptosis of breast cancer cells and its mechanism, Chinese Journal of Gerontology. 2019. 39(19): 4846-4850.

21. Rebecca Klee, Kathrin Töllner, Vladan Rankovic, et al. Network pharmacology for antiepileptogenesis: Tolerability of multitargeted drug combinations in nonepileptic vs. post-status epilepticus mice. Epilepsy Research, 2015, 118:34-48.

22. Liu HP, Zeng LT, Yang KL,et al. A Network Pharmacology Approach to Explore the Pharmacological Mechanism of Xiaoyao Powder on Anovulatory Infertility. Evidence-Based Complementary and Alternative Medicine,2016,2016.

23. Jiang Y, Zhou JP, Zhao JS , et al., MiR-18a-downregulated RORA inhibits the proliferation and tumorigenesis of glioma using the TNF-a-mediated NF-KB signaling pathway. EbioMedicine,2020. 52: 102651.

24. Huang QW, Huang T, Niu ML, et al. Linarin inhibits the migration and invasion of human breast cancer MDA-MB-231 cells by regulating IKK / NF - $\mathrm{K} B$ signaling pathway. Chinese Journal of pathophysiology, 2019, 035 (012): 2194-2200.

25. D, W.D., The role of TNF-a in the occurrence and development of gastric cancer and its research progress. J Intractable Dis, 2018: 17( 4) :428 ख32区.

26. Leva GD , Garofalo M , Croce CM . MicroRNAs in Cancer. Annual Review of Pathology, 2014, 9(1).

27. Benchetrit $F$, Ciree A, Vives $V$, et al. Interleukin-17 inhibits tumor cell growth by means of a T-celldependent mechanism. Blood, 2002, 99(6):2114-2121. 
28. Yu X, Ling J, Liu X, et al. Cordycepin induces autophagy-mediated c-FLIPL degradation and leads to apoptosis in human non-small cell lung cancer cells. Oncotarget, 2017, 8(4):6691.

29. Xin, Jiang, Tang, et al. Cordycepin Exerts Neuroprotective Effects via an Anti-Apoptotic Mechanism based on the Mitochondrial Pathway in a Rotenone-Induced Parkinsonism Rat Model. CNS \& neurological disorders drug targets, 2019, 18(8):609-620.

30. Laura, Lossi, Claudia, et al. Caspase-3 Mediated Cell Death in the Normal Development of the Mammalian Cerebellum. International Journal of Molecular Sciences, 2018.

\section{Figures}
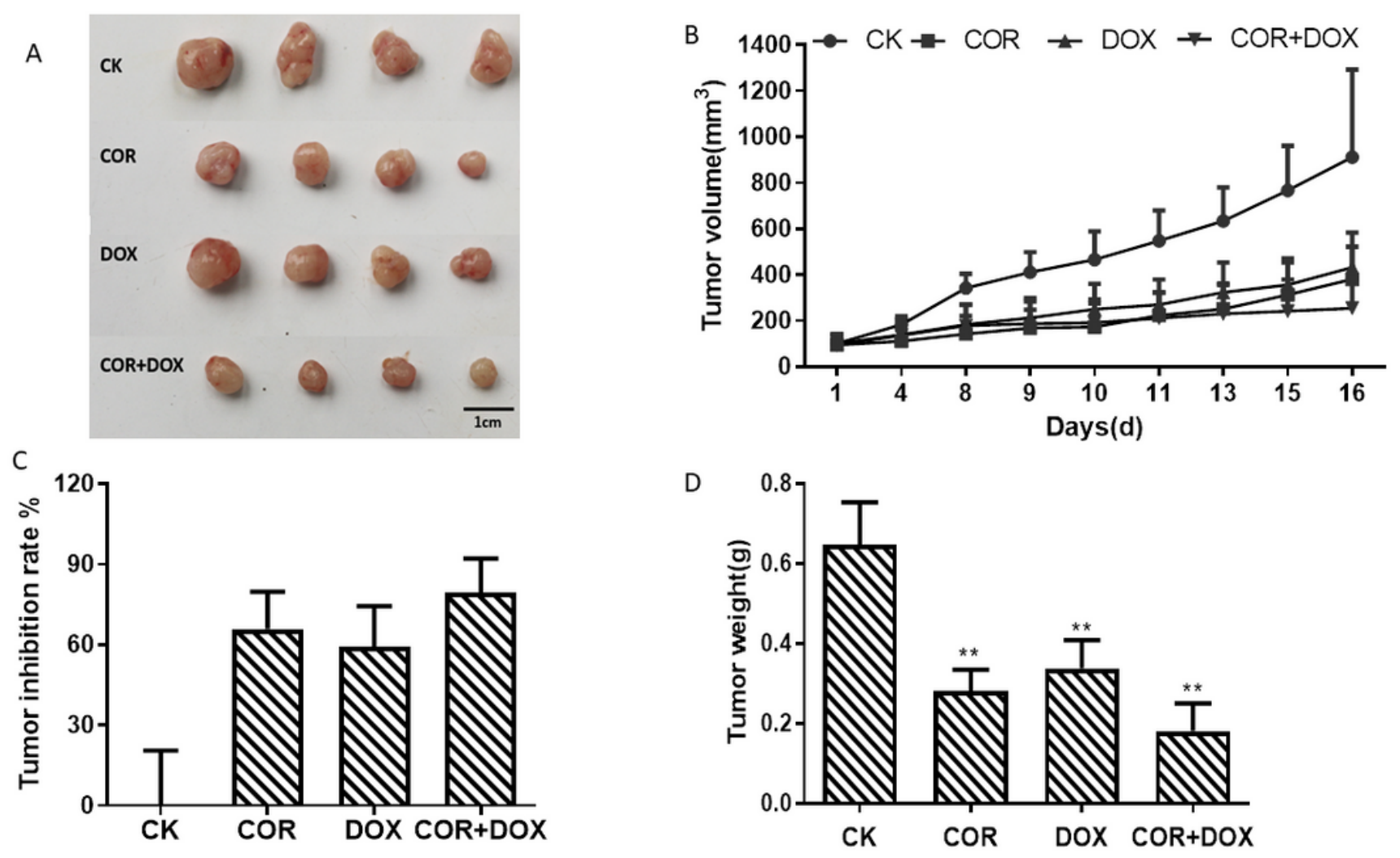

\section{Figure 1}

Effects of cordycepin combined with doxorubicin on xenograft tumor.(A) Effects of cordycepin combined with doxorubicin on tumor size; (B) Effects of cordycepin combined with doxorubicin on tumor volume; (C) Effects of cordycepin combined with doxorubicin on tumor inhibition; (D) Effects of cordycepin combined with doxorubicin on tumor weight. Compared with CK group * $p<0.05,{ }^{*} p<0.01$ 

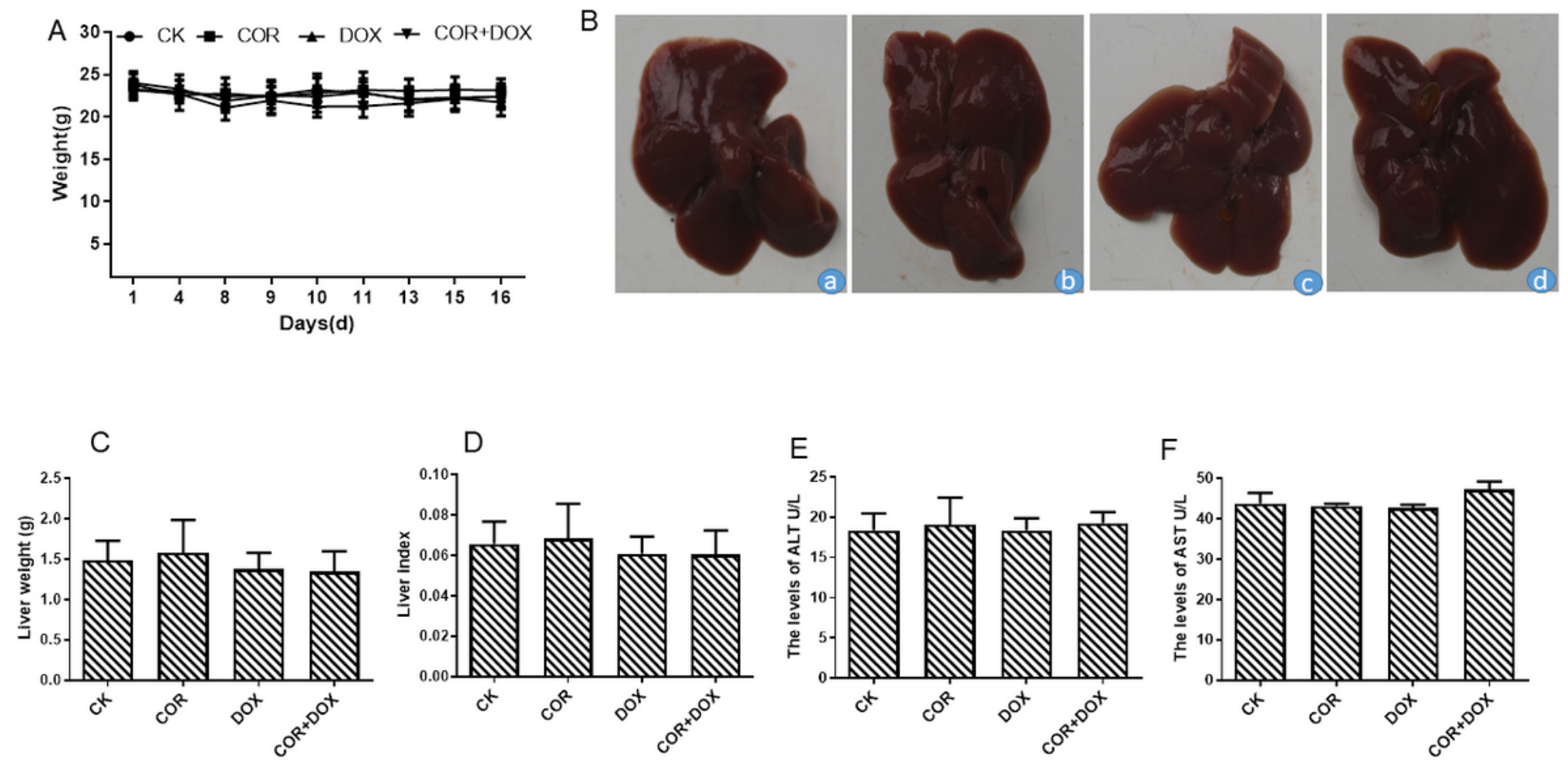

\section{Figure 2}

Toxic and Side Effects of Cordycepin combined with Doxorubicin on the of nude mice (A) The liver weight of nude mice . (B) The hepatic morphological of nude mice. a.Control group; b.COR group; c.DOX group; d.COR+DOX group. (C) The liver weight of nude mice. (D) The liver index of nude mice. (E) ALT level. (F) AST level. 

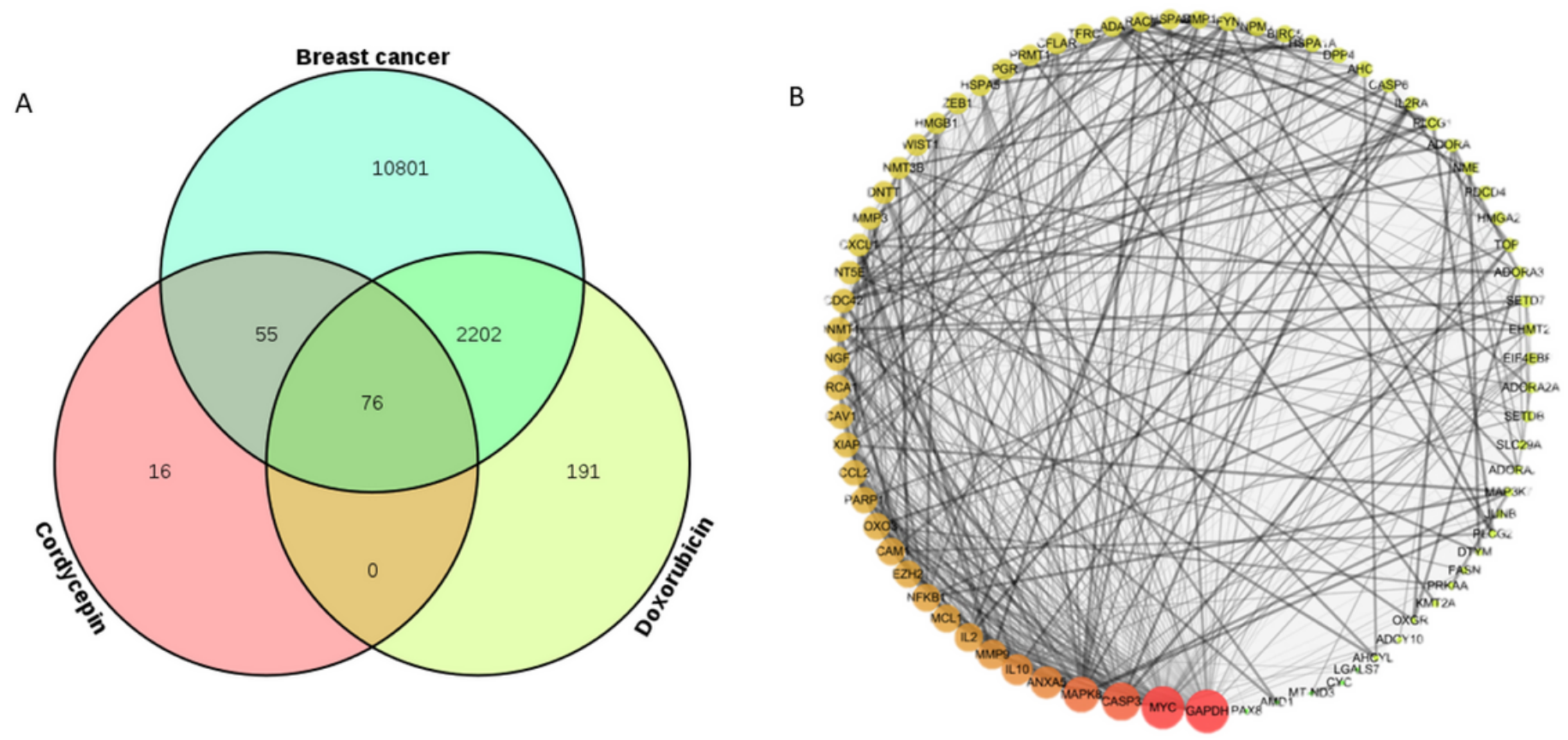

\section{Figure 3}

Screening and interaction of potential targets of cordycepin, doxorubicin and breast cancer.(A) potential targets of cordycepin ,doxorubicin, breast cancer . (B)Interaction network diagram of potential targets of cordycepin ,doxorubicin, breast cancer 
A

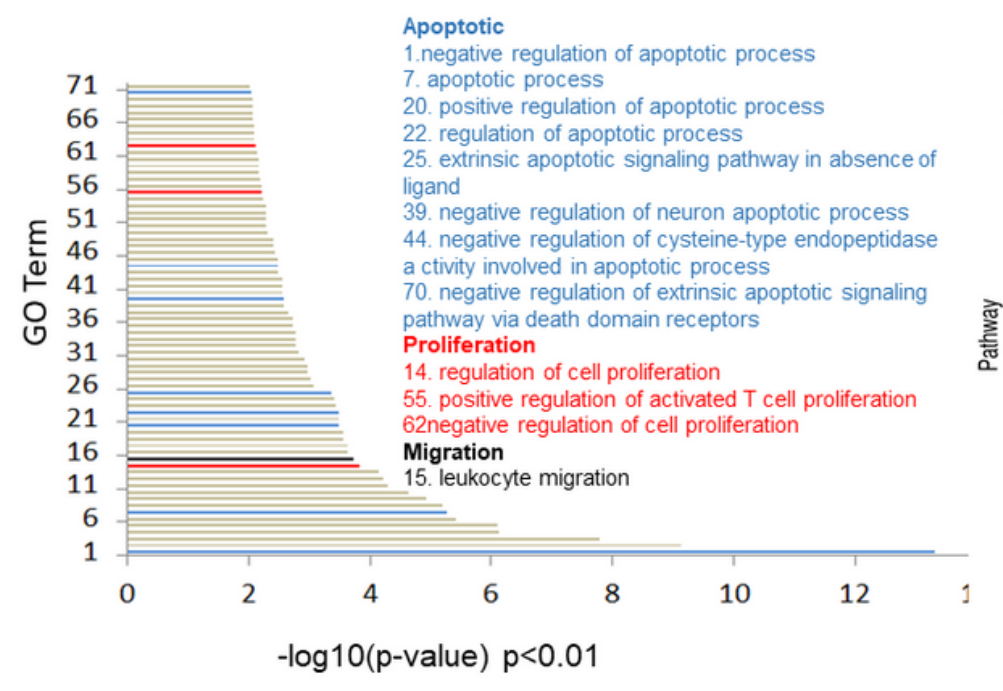

B

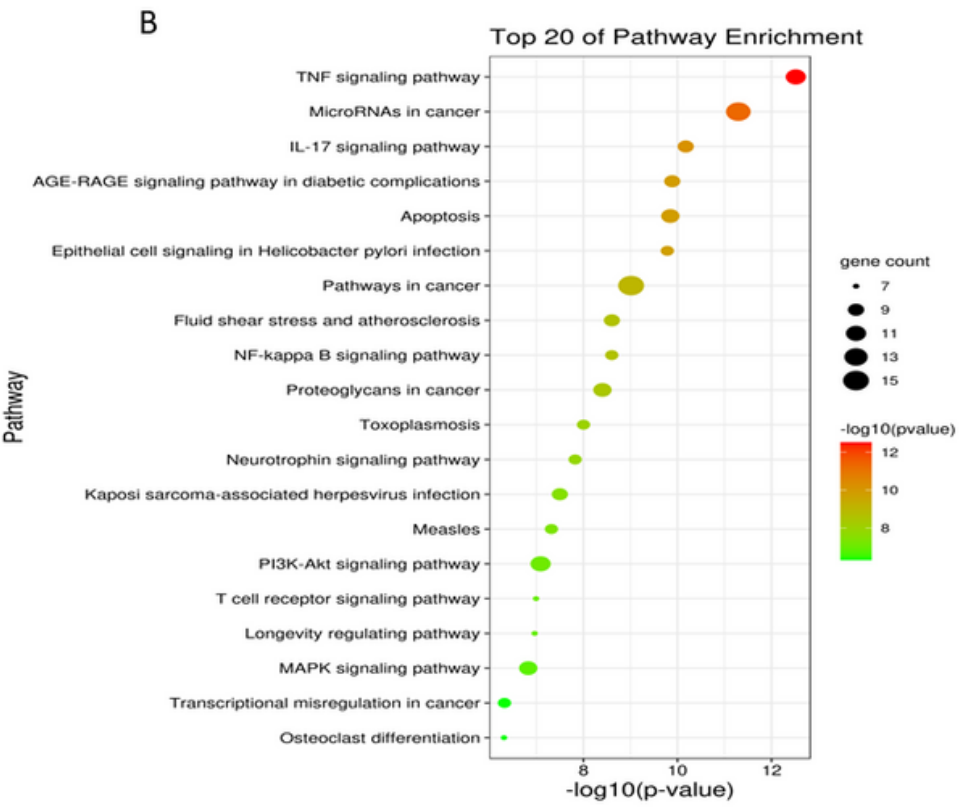

\section{Figure 4}

Biological function and pathway analysis of cordycepin combined with doxorubicin against breast cancer. (A) Biological processes of cordycepin combined with doxorubicin against breast cancer. The text on the right is the top 20 biological processes, the blue font is the biological process related to apoptosis, the red font is the biological process related to Proliferation, and the black font is the biological process related to migration. (B) Top 20 of pathway enrichment of cordycepin combined with doxorubicin against breast cancer. 


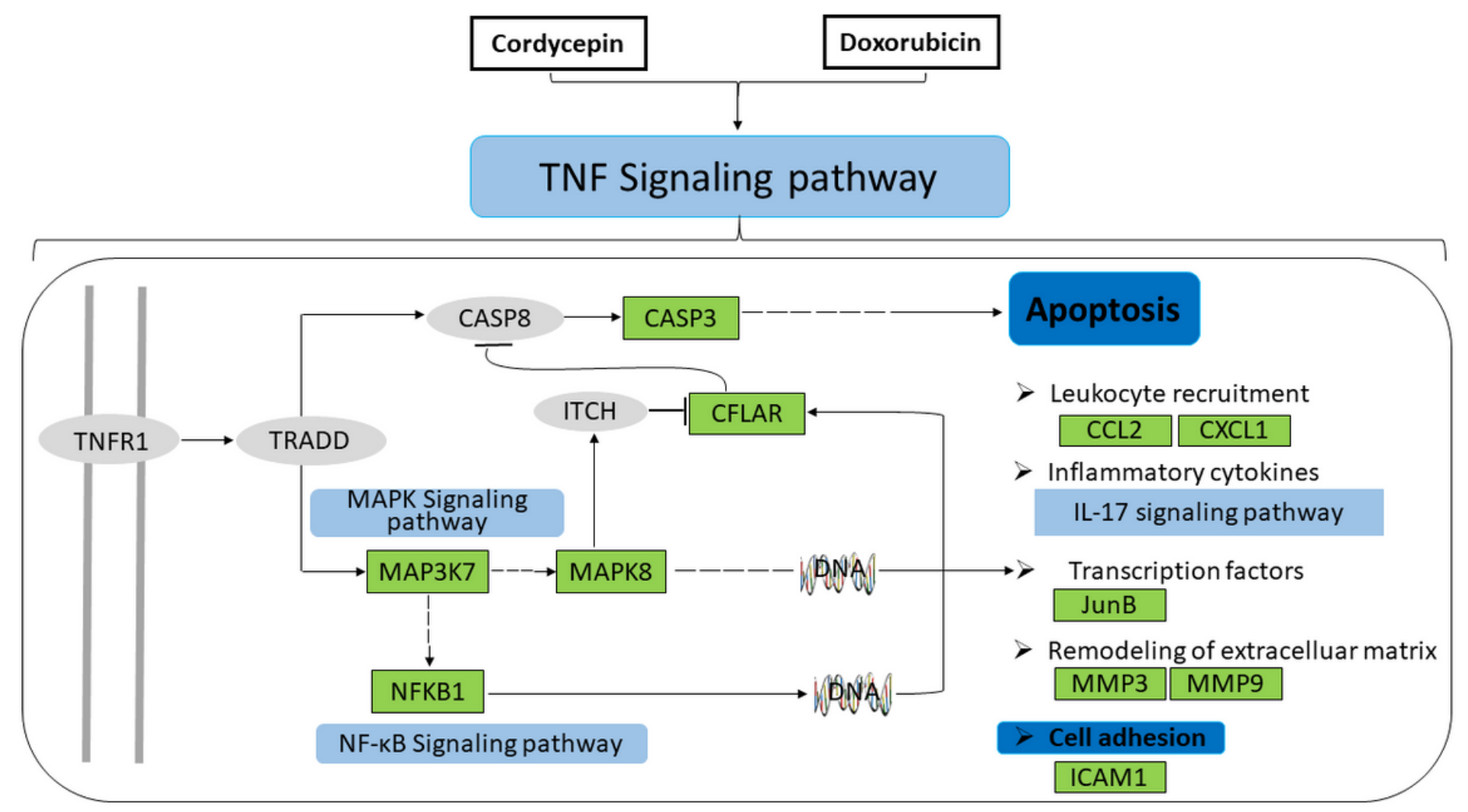

Figure 5

Potential targets pathway analysis of Cordycepin combined with Doxorubicin against breast cancer. Green indicates the predicted effective target, blue indicates the predicted effective biological process, light blue indicates the predicted effective signal pathway, gray indicates the potential target to be confirmed. 
A
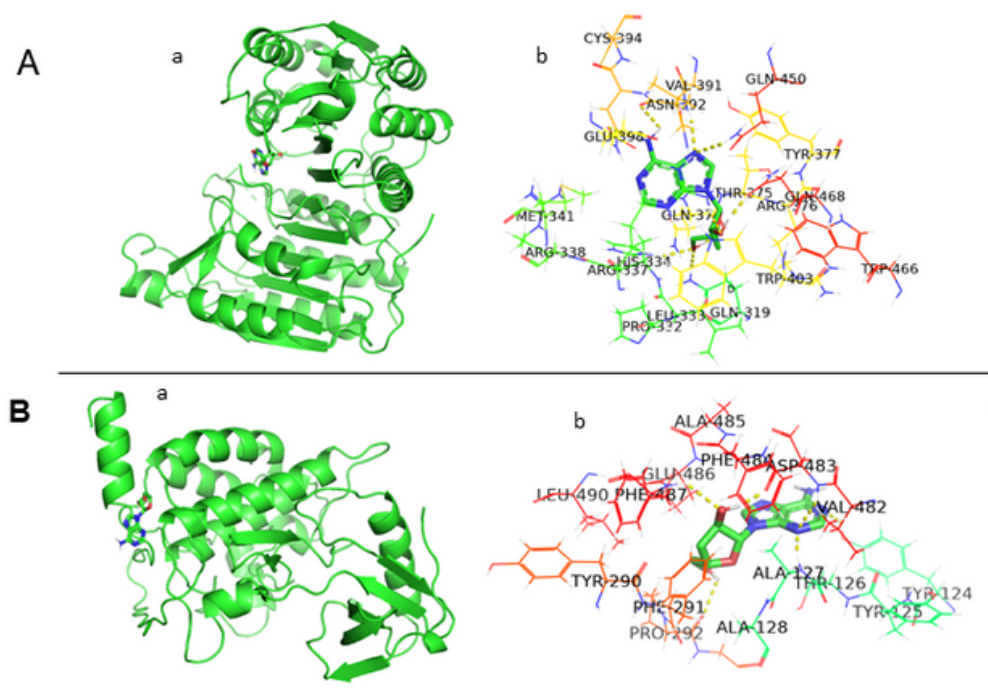

$c$

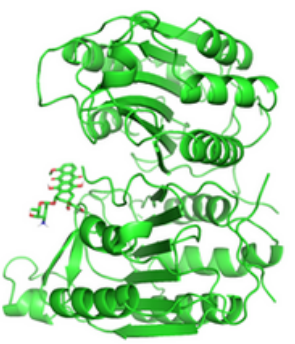

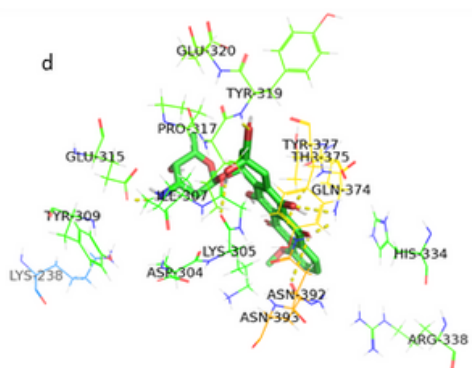
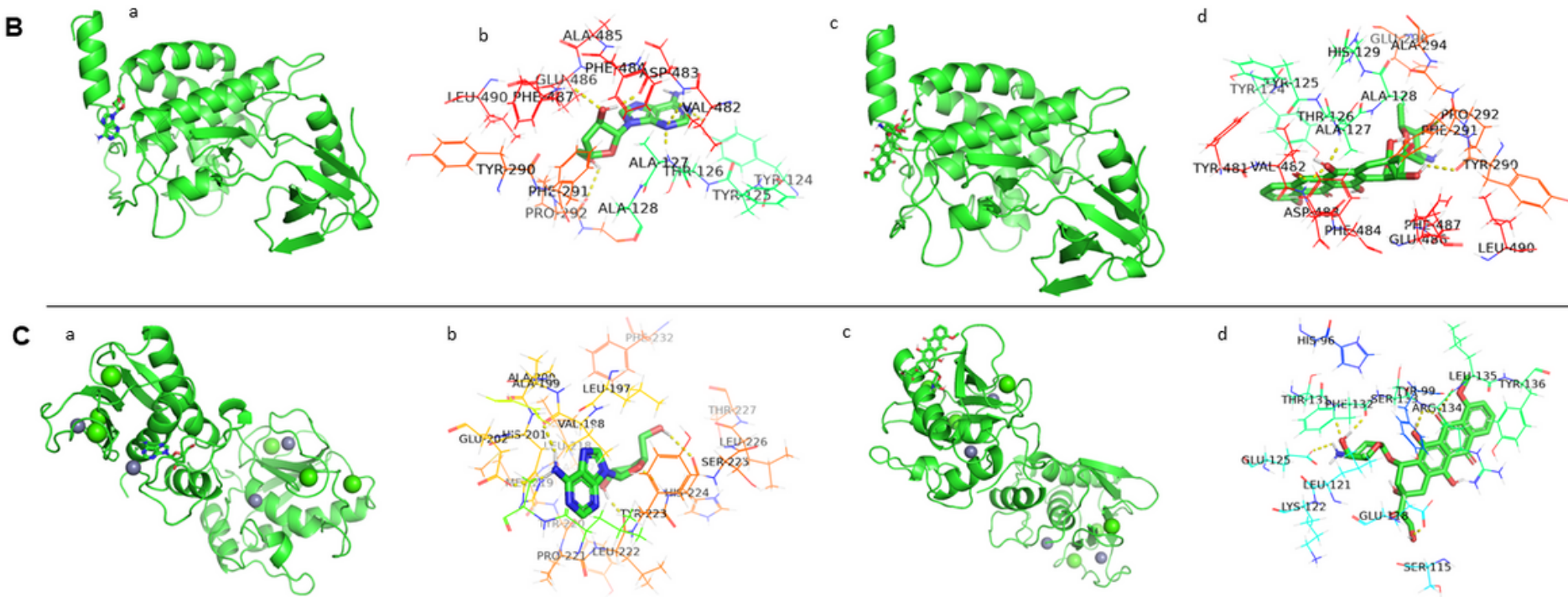

d

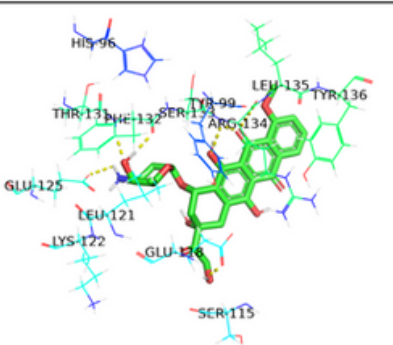

\section{Figure 6}

Docking of Cordycepin and Doxorubicin with CFLAR, MAP3K7 and MMP3 protein.(A).CFLAR. a-b Cordycepin docking with CFLAR;c-d. Doxorubicin docking with CFLAR .(B). MAP3K7. a-b Cordycepin docking with MAP3K7;c-d. Doxorubicin docking with MAP3K7.(C). MMP3. a-b Cordycepin docking with MMP3;c-d. Doxorubicin docking with MMP3. 

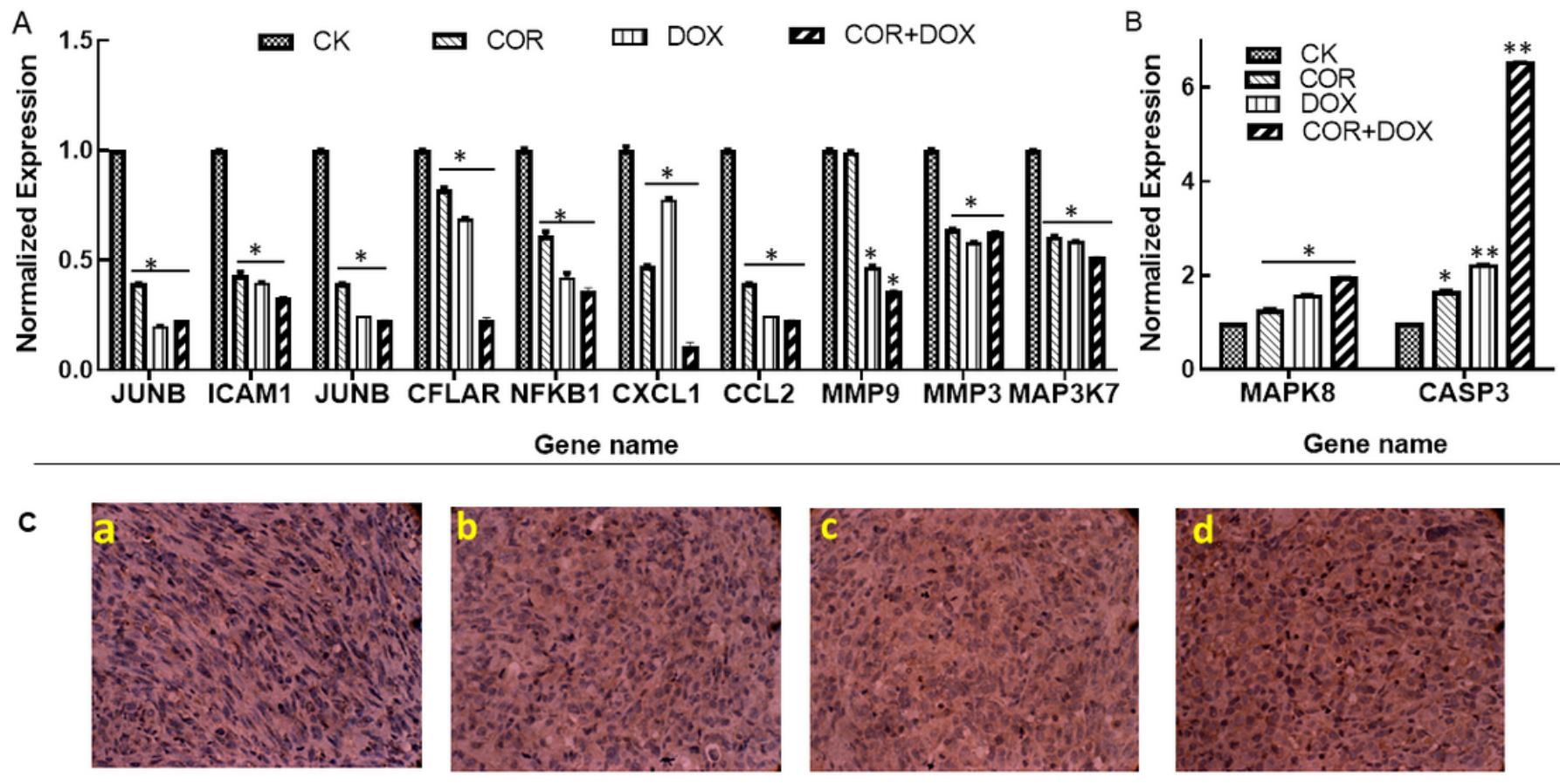

Figure 7

Expression analysis of targets of TNF signaling pathway in breast cancer(A-B).Gene expression. Compared with Control group ${ }^{*}<0.05,{ }^{\star *} p<0.01$.(C). The expression of CASP3 by immunohistochemical detection .a.Control group b.COR group c.DOX group d.COR+DOX group. Amplification factor $10 \times 20$. 